\title{
Editorial: Outstanding PRST-AB Articles from 2010
}

Under an initiative of PRST-AB Editorial Board Member Vladimir Shiltsev, six outstanding PRST-AB papers from the year 2010 have been selected and approved by the PRST-AB Editors. The selection criteria included the original referee assessments, the number of citations since publication, and the editors' own impression of a paper's importance. The selected PRST$\mathrm{AB}$ articles from 2010 provide a well balanced picture of PRST-AB coverage:

1. Z. Huang, A. Brachmann, F.-J. Decker, Y. Ding, D. Dowell, P. Emma, J. Frisch, S. Gilevich, G. Hays, Ph. Hering, R. Iverson, H. Loos, A. Miahnahri, H.-D. Nuhn, D. Ratner, G. Stupakov, J. Turner, J. Welch, W. White, J. Wu, and D. Xiang, Measurements of the linac coherent light source laser heater and its impact on the x-ray free-electron laser performance, Phys. Rev. ST Accel. Beams 13, 020703 (2010).

2. Christopher M. S. Sears, Alexander Buck, Karl Schmid, Julia Mikhailova, Ferenc Krausz, and Laszlo Veisz, Emittance and divergence of laser wakefield accelerated electrons, Phys. Rev. ST Accel. Beams 13, 092803 (2010).

3. F. Stephan, C. H. Boulware, M. Krasilnikov, J. Bähr, G. Asova, A. Donat, U. Gensch, H. J. Grabosch, M. Hänel, L. Hakobyan, H. Henschel, Y. Ivanisenko, L. Jachmann, S. Khodyachykh, M. Khojoyan, W. Köhler, S. Korepanov, G. Koss, A. Kretzschmann, H. Leich, H. Lüdecke, A. Meissner, A. Oppelt, B. Petrosyan, M. Pohl, S. Riemann, S. Rimjaem, M. Sachwitz, B. Schöneich, T. Scholz, H. Schulze, J. Schultze, U. Schwendicke, A. Shapovalov, R. Spesyvtsev, L. Staykov, F. Tonisch, T. Walter, S. Weisse, R. Wenndorff, M. Winde, L. v. Vu, H. Dürr, T. Kamps, D. Richter, M. Sperling, R. Ovsyannikov, A. Vollmer, J. Knobloch, E. Jaeschke, J. Boster, R. Brinkmann, S. Choroba, K. Flechsenhar, K. Flöttmann, W. Gerdau, V. Katalev, W. Koprek, S. Lederer, C. Martens, P. Pucyk, S. Schreiber, S. Simrock, E. Vogel, V. Vogel, K. Rosbach, I. Bonev, I. Tsakov, P. Michelato, L. Monaco, C. Pagani, D. Sertore, T. Garvey, I. Will, I. Templin, W. Sandner, W. Ackermann, E. Arévalo, E. Gjonaj, W. F. O. Müller, S. Schnepp, T. Weiland, F. Wolfheimer, J. Rönsch, and J. Rossbach, Detailed characterization of electron sources yielding first demonstration of European X-ray Free-Electron Laser beam quality, Phys. Rev. ST Accel. Beams 13, 020704 (2010).

4. V. Danilov and S. Nagaitsev, Nonlinear accelerator lattices with one and two analytic invariants, Phys. Rev. ST Accel. Beams 13, 084002 (2010).

5. Yuantao Ding, Zhirong Huang, and Ronald D. Ruth, Two-bunch self-seeding for narrowbandwidth hard x-ray free-electron lasers, Phys. Rev. ST Accel. Beams 13, 060703 (2010).

6. K. R. LeChien, W. A. Stygar, M. E. Savage, P. E. Wakeland, V. Anaya, D. S. Artery, M. J. Baremore, D. E. Bliss, R. Chavez, G. D. Coombs, J. P. Corley, P. A. Jones, A. K. Kipp, B. A. Lewis, J. A. Lott, J. J. Lynch, G. R. McKee, S. D. Ploor, K. R. Prestwich, S. A. Roznowski, D. C. Spencer, S. D. White, and J. R. Woodworth, 6.1-MV, 0.79-MA laser-triggered gas switch for multimodule, multiterawatt pulsed-power accelerators, Phys. Rev. ST Accel. Beams 13, 030401 (2010).

This identification of six key articles from 2010 represents a first step towards bringing important papers to the attention of our readers. Improved mechanisms and modes for highlighting outstanding PRST-AB articles are presently under development.

Frank Zimmermann

Editor

Published 17 January 2012

DOI: 10.1103/PhysRevSTAB.15.010001

PACS numbers: 01.30.Ww

Published by the American Physical Society under the terms of the Creative Commons Attribution 3.0 License. Further distribution of this work must maintain attribution to the author(s) and the published article's title, journal citation, and DOI. 